\section{Remote supervision and fault detection on OPC monitored PV systems}

\section{Santiago Silvestre ${ }^{\mathrm{a}, *}$}

Santiago.silvestre@upc.edu

\section{Llanos Mora-López}

llanos@lcc.uma.es

Sofiane

kichousofiane@gmail.com

Francisco

fsanchezp@uma.es

Manue

Manuel Dominguez@upc.edu
๑2016. This manuscript version is made available under the CC-BY-NC-ND 4.0 license http://creativecommons.org/ licenses/by-ne-nd $/ 4.0 /$

DOI: $10.1016 / j$. solener.2016.08.030

aMNT Group, Electronic Engineering Department, UPC-BarcelonaTech, Barcelona, C/ Jordi Girona 1-3, Mòdul C4 Campus Nord UPC, 08034 Barcelona, Spain

bDpto. Lenguajes y Ciencias de la Computación, Universidad de Málaga, Campus de Teatinos, sn, Málaga 29071, Spain

'Dpto. Tecnología Eléctrica, Universidad de Málaga, Campus de Teatinos, sn, Málaga 29071, Spain

${ }^{*}$ Corresponding author

\title{
Abstract
}

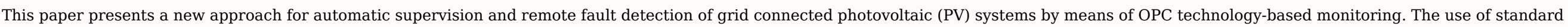

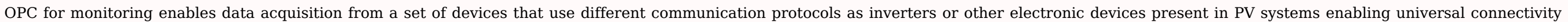

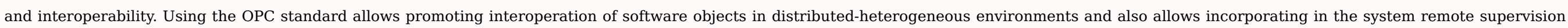

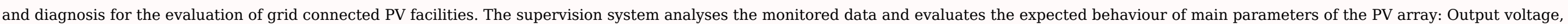

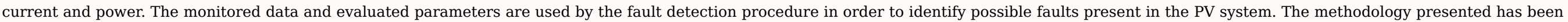

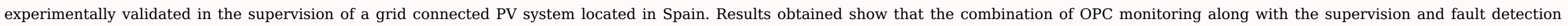

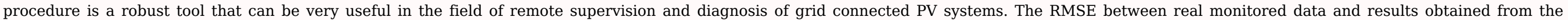
modelling of the PV array were below $3.6 \%$ for all parameters even in cloudy days.

Keywords: OPC; Monitoring; Fault detection; PV systems

\section{Introduction}

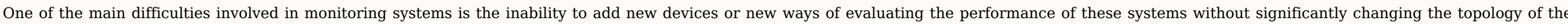

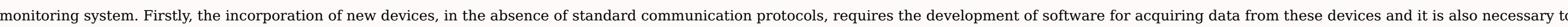

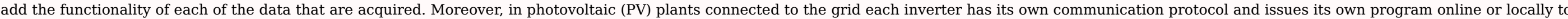

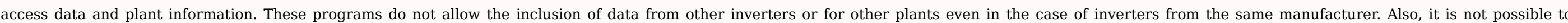




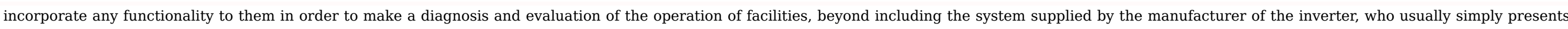

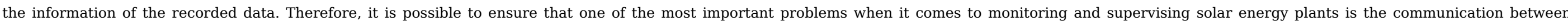

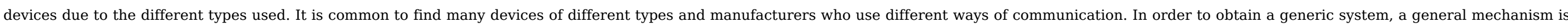
needed to communicate with any devices, irrespective of their characteristics or of the manufacturer.

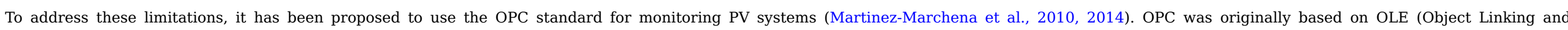

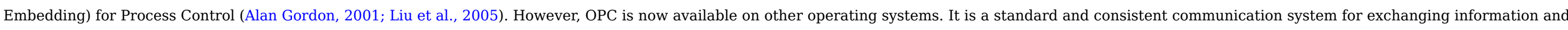

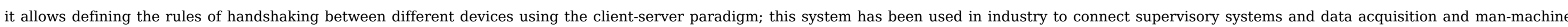

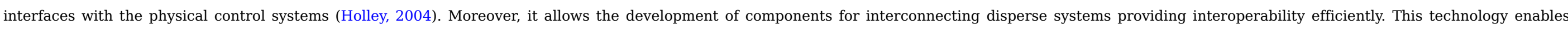
software components developed by experts in one sector to be used by applications in any other sector. The design of OPC interfaces supports distributed architectures.

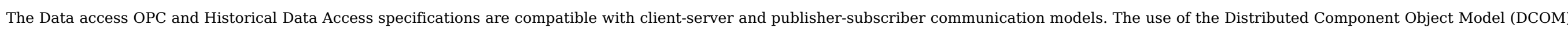

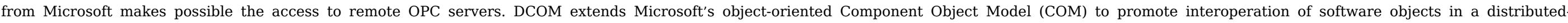
heterogeneous environment.

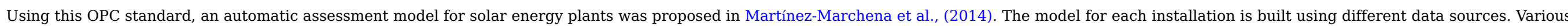
daily parameters were proposed to evaluate the performance of a photovoltaic system:

- The daily output energy of the photovoltaic plant, that is, the daily energy supplied by the installation, $\mathrm{E}_{\text {day }}$.

- The daily yield, $\mathrm{Y}_{\mathrm{a} \_ \text {day, }}$ defined as the daily output energy per $\mathrm{kW}_{\mathrm{p}}$ installed.

The daily evaluation model is treated as an element of the system. The container used for the model behaves as an OPC client with access to all data.

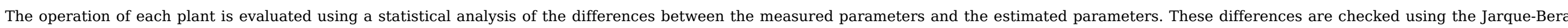

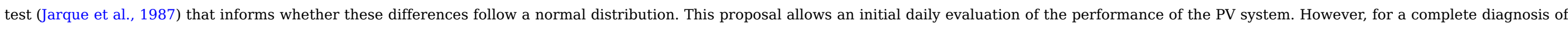
the detected problems generally related to the DC side of the PV system, it is necessary to use additional methods based on a detailed analysis of monitored data.

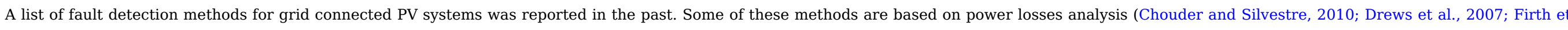

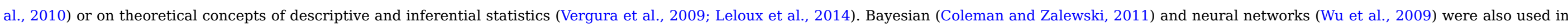

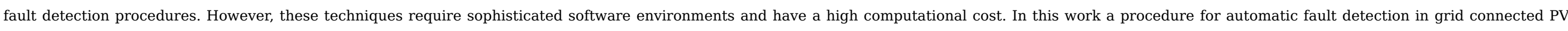

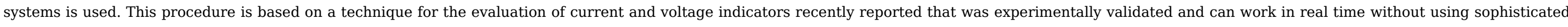

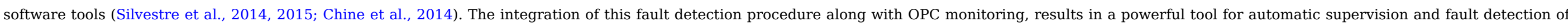

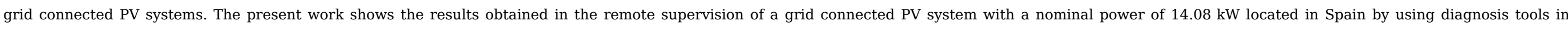
combination with OPC monitoring.

\section{Methodology}

\subsection{Description of the OPC-based monitoring}

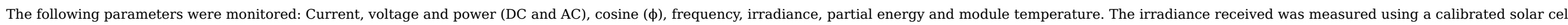

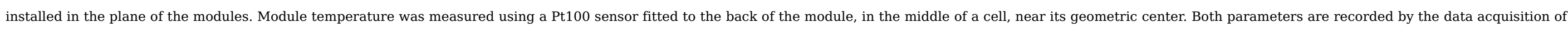
the inverter.

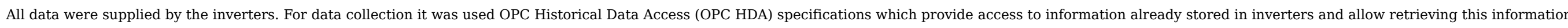




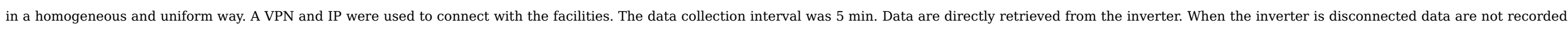
but data previously stored in the inverter will be transmitted when the inverter is connected.

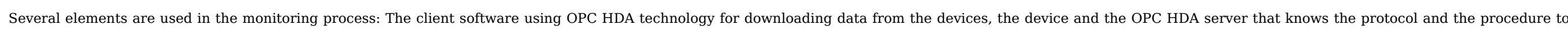
download data from the device (Martínez-Marchena, 2015).

Data were stored in a PostgreSQL DBMS compatible with the SQL92 standard. Daily evaluation and fault detection algorithms were implemented with OPC.

\subsection{PV system modelling}

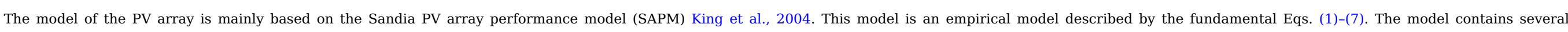

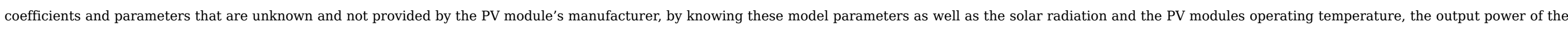
$\mathrm{PV}$ array can be predicted by using the following equations:

$$
E e=G / G_{n}
$$$$
I s c g=N_{p g}\left[I s c o \cdot E e \cdot\left\{1+\alpha_{I s c} \cdot(T c-T o)\right\}\right]
$$$$
\text { Impg }=N_{p g}\left[\operatorname{Impo} \cdot\left\{C_{0} \cdot E e+C_{1} \cdot E e^{2}\right\} \cdot\left\{1+\alpha_{I m p} \cdot(T c-T o)\right\}\right]
$$$$
\delta(T c)=n \cdot k \cdot(T c+273.15) / q
$$$$
\text { Vocg }=N_{s g}\left[\operatorname{Voco}+N_{s} \cdot \delta(T c) \cdot \ln (E e)+\beta_{V o c}(E e) \cdot(T c-T o)\right]
$$$$
V m p g=N_{s g}\left[V m p o+C_{2} \cdot N_{s} \cdot \delta(T c) \cdot \ln (E e)+C_{3} \cdot N_{s} \cdot\{\delta(T c) \cdot \ln (E e)\}^{2}+\beta_{V m p}(E e) \cdot(T c-T o)\right]
$$

Pmpg $=$ Impg. Vmpg

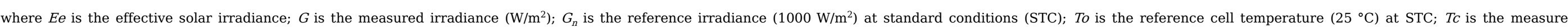

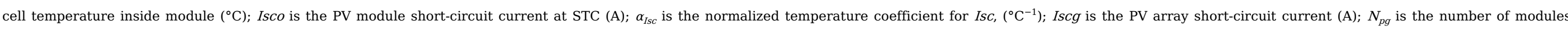

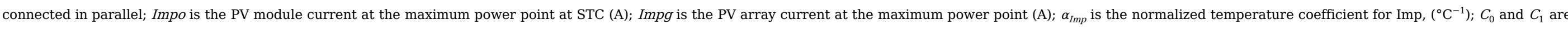

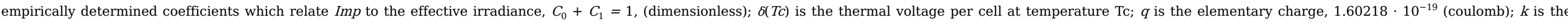

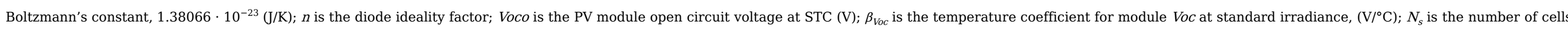

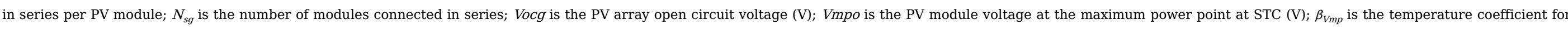

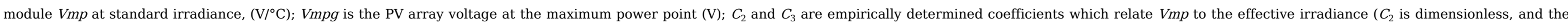
unit of $C_{3}$ is $\left(\mathrm{V}^{-1}\right)$ and finally $P m p$ is the $\mathrm{PV}$ array power at the maximum power point (W).

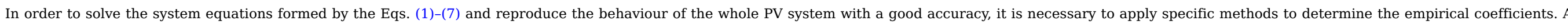

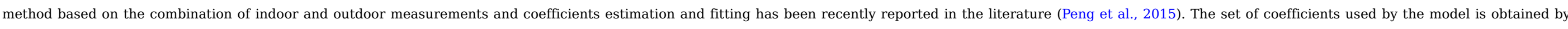

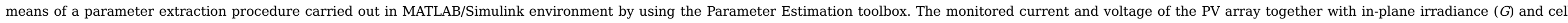
temperature $(T C)$ profiles are needed to estimate the set of unknown parameters of SAPM model implemented in Simulink as illustrated in Fig. 1. 


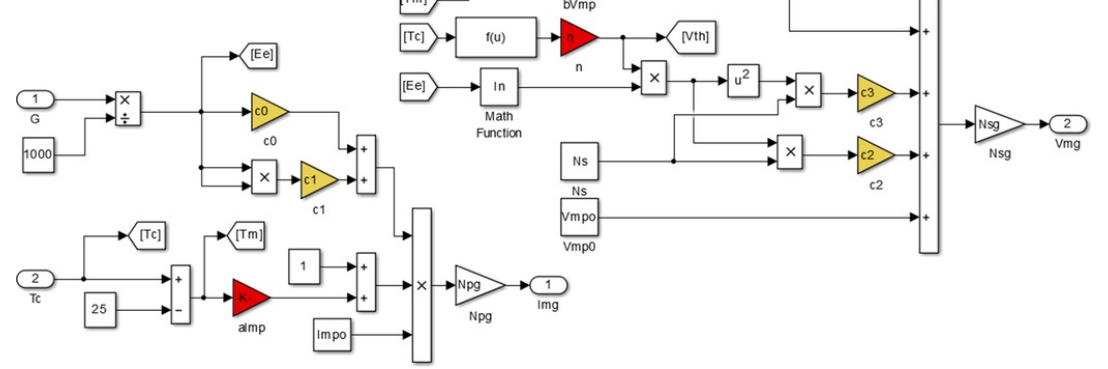

Fig. 1 Simulink block diagram of the parameter extraction algorithm.

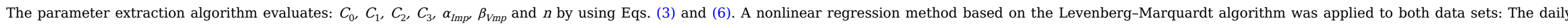

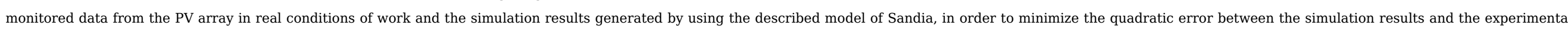
data.

\subsection{Fault detection procedure}

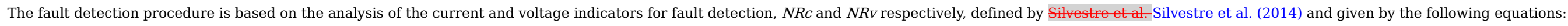
$N R c=\frac{I_{m}}{I_{s c g}}$ $N R v=\frac{V_{m}}{V_{o c g}}$

where Vm and Im are the coordinates of the maximum power point (MPP) at the DC side of the PV array.

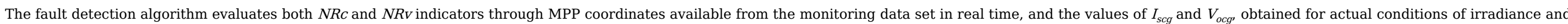

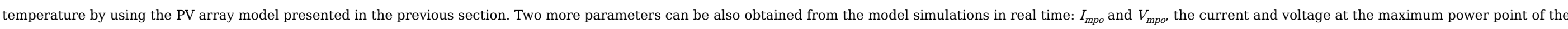
output of the PV array in absence of faults and normal operation of the PV array (Silvestre et al., 2014). Then, the expected values of $N R c$ and $N R v$ : NRco and $N R v o$, are given by:

$N R c o=\frac{I_{m p o}}{I_{s c g}}$

$N R v o=\frac{V_{m o}}{V_{o c g}}$

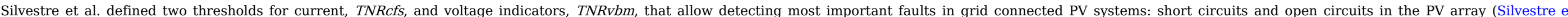
al., 2014) as well as inverter disconnection or partial shading conditions of work (Silvestre et al., 2015). These thresholds were defined by the following equations:

TNRcfs $=1.02 \alpha N$ Rco

$T N R v b m=1.02 \beta N R c o$

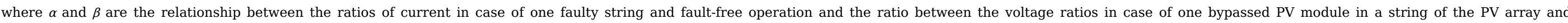
fault-free operation respectively (Silvestre et al., 2014, 2015).

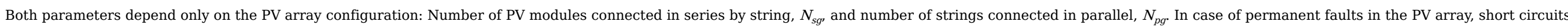

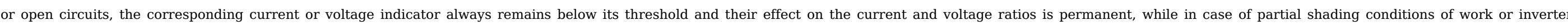

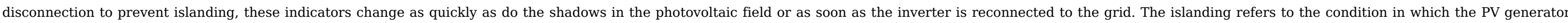

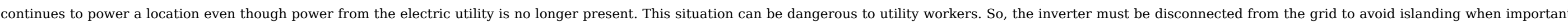




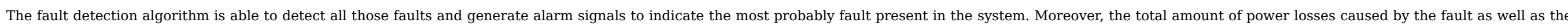

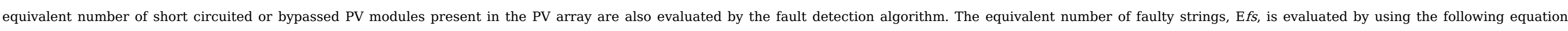
(Silvestre et al., 2015):

$E f s=N p g\left(1-\frac{N R c}{N R c o}\right)$

Finally, the number of equivalent bypassed modules, $B P \mathrm{mod}$, present on the PV array is estimated as follows:

$B P \bmod =N s g\left(1-\frac{N R v}{N R v o}\right)$

The proportion of DC power losses due to the shadowing effect, Ploss, is also evaluated by the automatic supervision procedure by using the following equation: Ploss $=\left(1-\frac{N R c}{N R c o} \frac{N R v}{N R v o}\right)$

The efficiency parameters used for the energetic evaluation of the system are the performance ratio $(P R)$ and the array yield $\left(Y_{a}\right)$ given by the following equations:

$Y_{a}=\frac{\int_{0}^{\Delta t} P_{a c} d t}{P_{o}}$

where $P_{a c}$ is the output power of the PV array and $P_{o}$ is the nominal power of the array.

$P R=\frac{Y a}{Y r}$

where $Y r$, is the daily total irradiation $\mathrm{H}$ in the array plane divided by the reference daily irradiance at STC.

\section{Results and discussion}

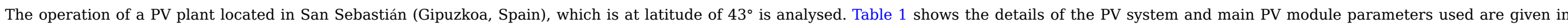
Table 2.

Table 1 PV system description.

\begin{tabular}{|c|c|}
\hline Main parameters & PV system \\
\hline PV module & IS 160 \\
\hline Nominal power & $14.08 \mathrm{kWp}$ \\
\hline Number of inverters & 3 \\
\hline Modules per inverter & $28 / 30 / 30$ \\
\hline Modules in series $(N s g)$ & $14 / 15 / 15$ \\
\hline Strings in parallel $(N p g)$ & $2 / 2 / 2$ \\
\hline Tilt & $20^{\circ}$ \\
\hline Orientation & $9^{\circ}$ East \\
\hline Inverters & Ingecon SUN 5 Single-phase inverter \\
\hline Inverters nominal power & $5 \mathrm{kWp}$ \\
\hline
\end{tabular}


Table 2 Main parameters of PV modules.

$$
\text { Isc (A) }
$$

Voc (V)

Current at maximum power point: Impp (A)

Voltage at maximum power point: Vmpp (V)

Temperature coefficient of Voc: $\beta_{\text {Voc }}\left(\mathrm{V} /{ }^{\circ} \mathrm{C}\right)$

Temperature coefficient of Isc: $\alpha_{I s c}\left(\mathrm{~A} /{ }^{\circ} \mathrm{C}\right)$

22.2

8.65

18.5

$-0.084$

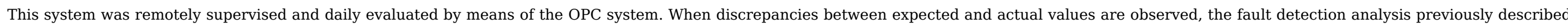
is applied. This analysis was carried out for the month of December 2014.

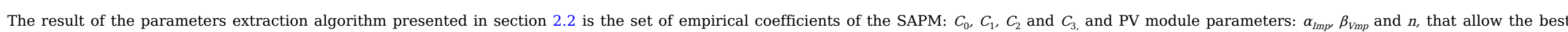

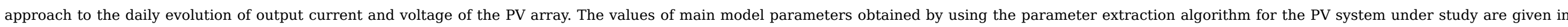
Table 3.

Table 3 Values obtained for model parameters.

$C_{0} \quad C_{1}$

$C_{1}\left(\mathrm{~V}^{-1}\right)$

$C_{3}$

$\alpha_{\operatorname{Imp}}\left(1 /{ }^{\circ} \mathrm{C}\right)$

$\beta_{V m p}\left(\mathrm{~V} /{ }^{\circ} \mathrm{C}\right)$

$n$

0.90336

0.002202

3.8319

99.94

$3.76810^{-4}$

$-0.10447$

1.1003

Figs. 2 and 3 illustrate the daily monitored profiles of irradiance and cell temperature of the PV array used as input data for the parameter extraction algorithm.

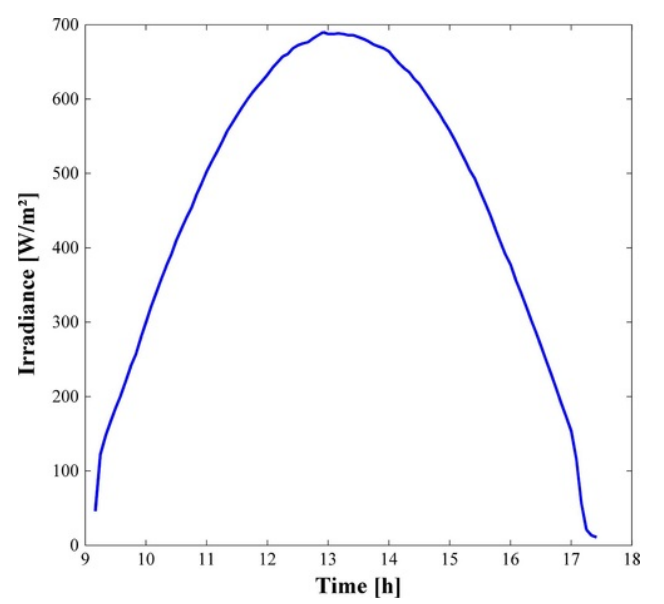

Fig. 2 Irradiance profile corresponding to 10th of December, 2014. 


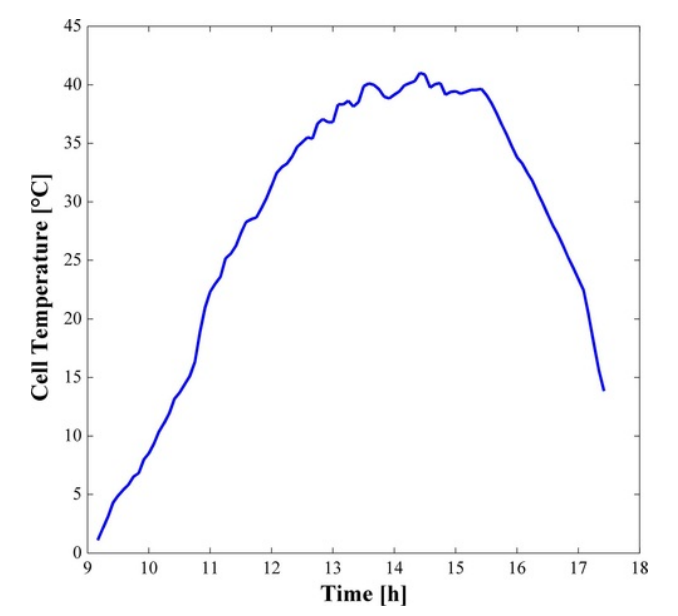

Fig. 3 Cell temperature corresponding to 10th of December, 2014.

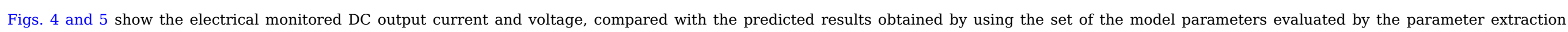
algorithm. The DC output power of the PV array is obtained as a product of current and voltage in both real and simulated results and the obtained result is illustrated in Fig. 6.

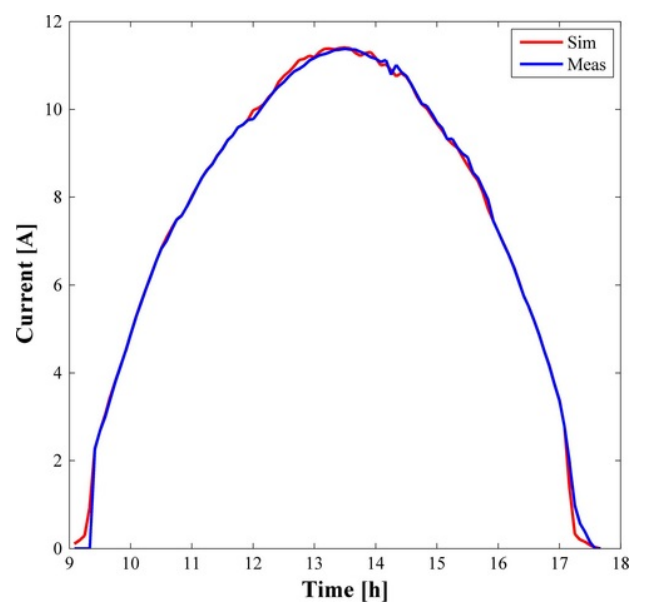

Fig. 4 Simulated and measured DC output Current corresponding to 10th of December, 2014. 


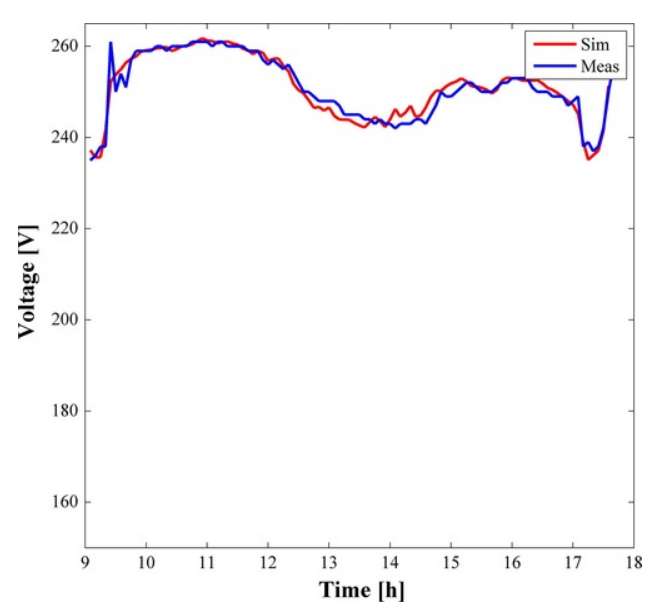

Fig. 5 Simulated and measured DC output voltage corresponding to 10th of December, 2014.

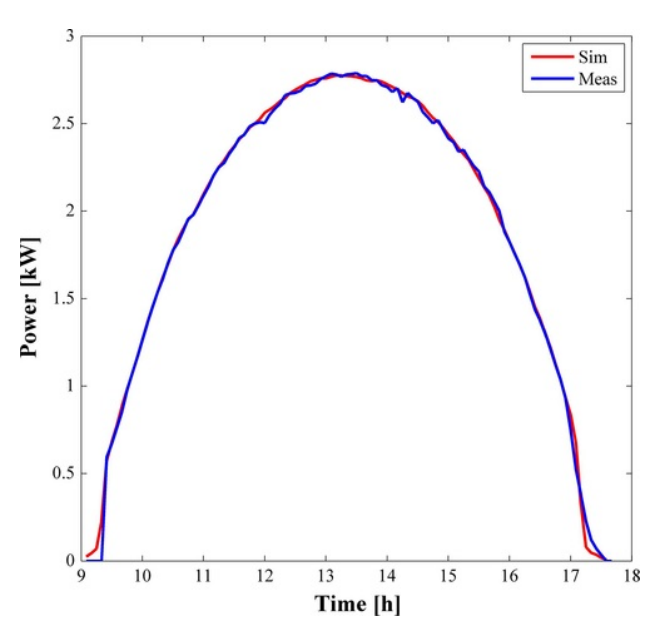

Fig. 6 Simulated and measured DC output Power corresponding to 10th of December, 2014.

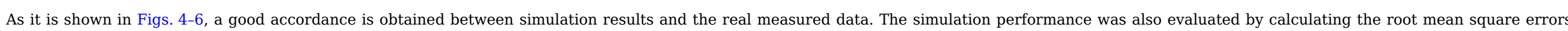
(RMSEs) of current, voltage and power between both data sets for different days with different climatic conditions.

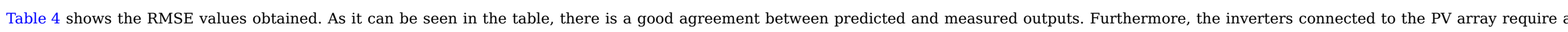

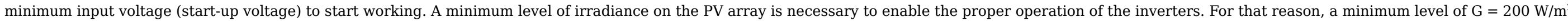

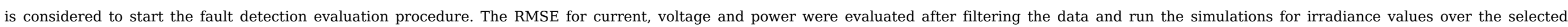
threshold of $200 \mathrm{~W} / \mathrm{m}^{2}$.

Table 4 Obtained RMSE (\%) for different weather conditions.

\begin{tabular}{|c|c|c|c|}
\hline Days & RMSE current (\%) & RMSE voltage (\%) & RMSE power (\%) \\
\hline Clear sky day $(G>=200)$ & 0.635 & 1.229 & 0.677 \\
\hline
\end{tabular}




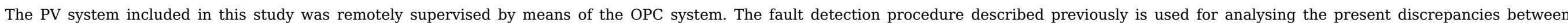
expected and actual values of the monitored parameters.

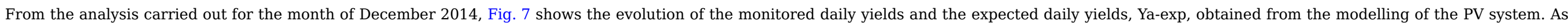

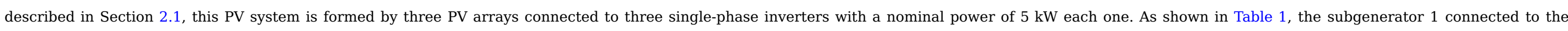
inverter 1 has 14 PV modules per string instead of 15. So, the subgenerator 1 has two PV modules least in the PV field that the other inverters.

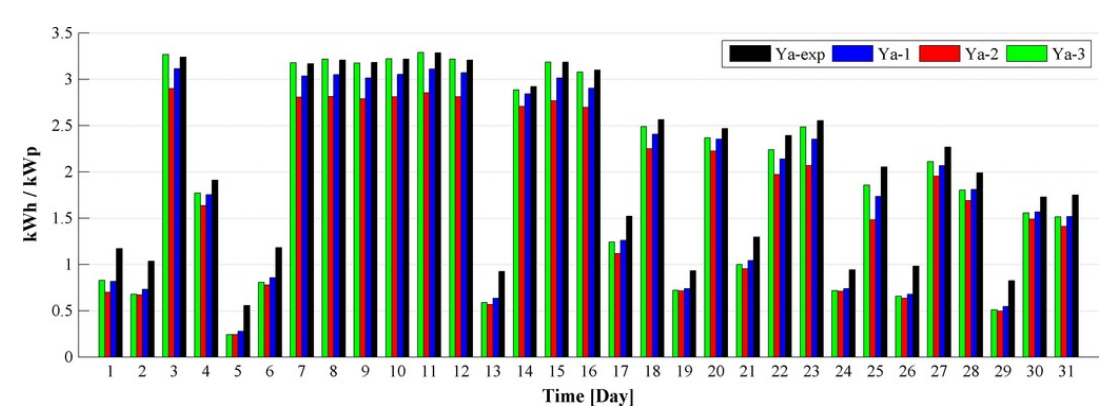

Fig. 7 Daily array yields corresponding to December 2014.

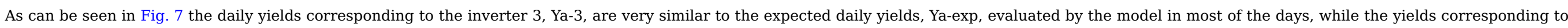
inverters 1 and 2, Ya-1 and Ya-2 respectively, are lower than Ya-3 and Ya-exp. Furthermore the sub-generator connected to the inverter 2 presents the lowest yield in all the days of the month.

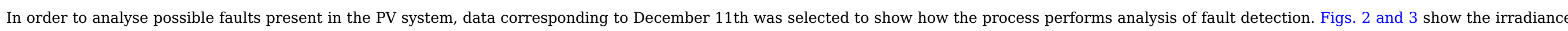
and temperature profiles measured on this day of December.

Table 5 shows the daily energy generated by each sub-generator of the PV system (DC and AC) as well as the performance ratio ( $P R)$.

Table 5 Values of the $P R$ and energy generated by the PV system 11th of December, 2014.

\begin{tabular}{|c|c|c|c|}
\hline & Daily $P R(\%)$ & Daily DC energy (kW h) & Daily AC energy ( $\mathrm{kW} \mathrm{h}$ ) \\
\hline Sub-generator 1 & 73.03 & 13.928 & 13.026 \\
\hline Sub-generator 2 & 66.96 & 13.683 & 12.797 \\
\hline Sub-generator 3 & 76.44 & 16.366 & 14.609 \\
\hline
\end{tabular}

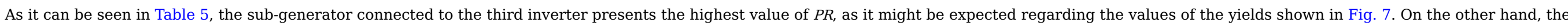

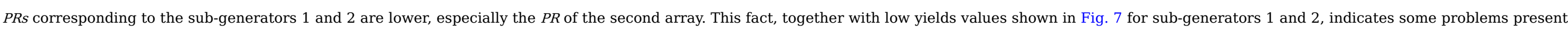
in the PV arrays in this time period. It is necessary to study the evolution of current and voltage indicators to identify the cause of these problems.

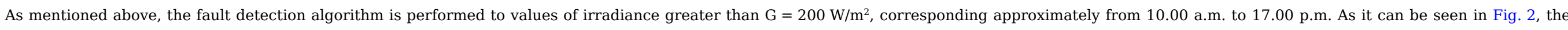

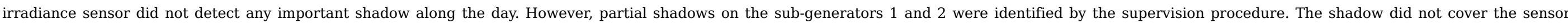
irradiance, but a part of the PV generator was affected. 


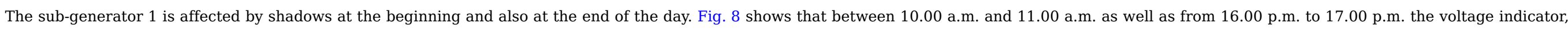

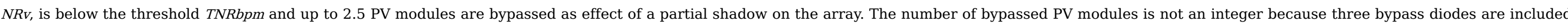

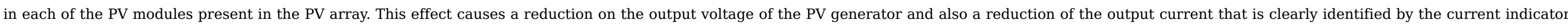

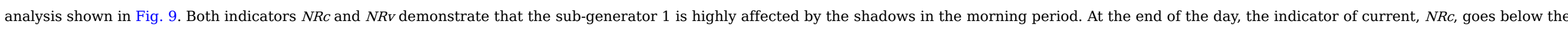
threshold, TNRcfs, and the reduction in output current is equivalent to one faulty string in this sub-generator.
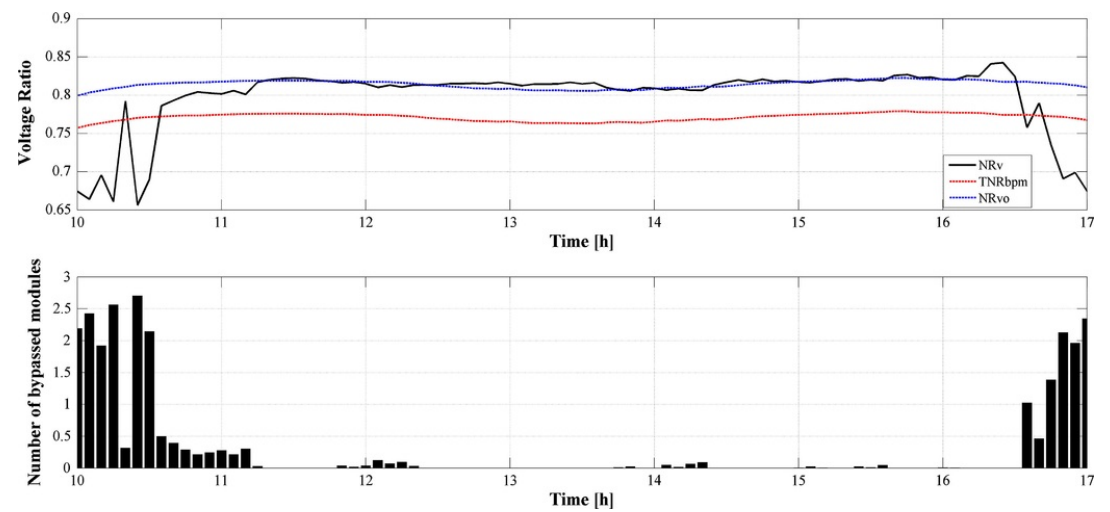

Fig. 8 Sub-generator 1. Evolution of the Voltage ratios and number of bypassed modules.
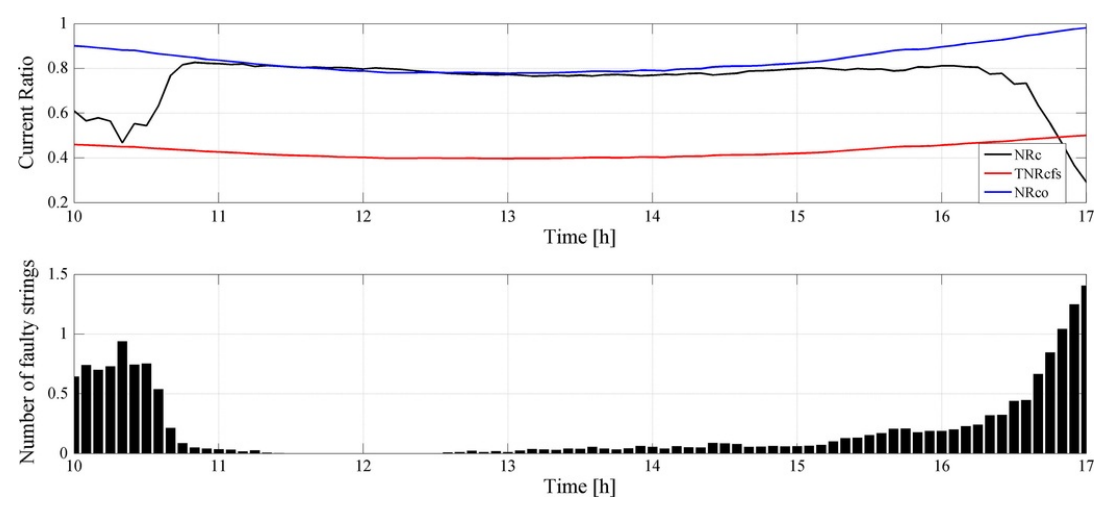

Fig. 9 Sub-generator 1: Evolution of the Current Ratios and equivalent number of faulty strings.

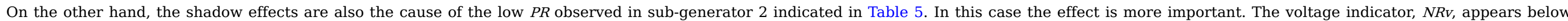

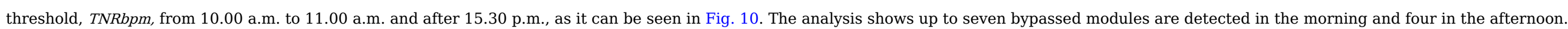



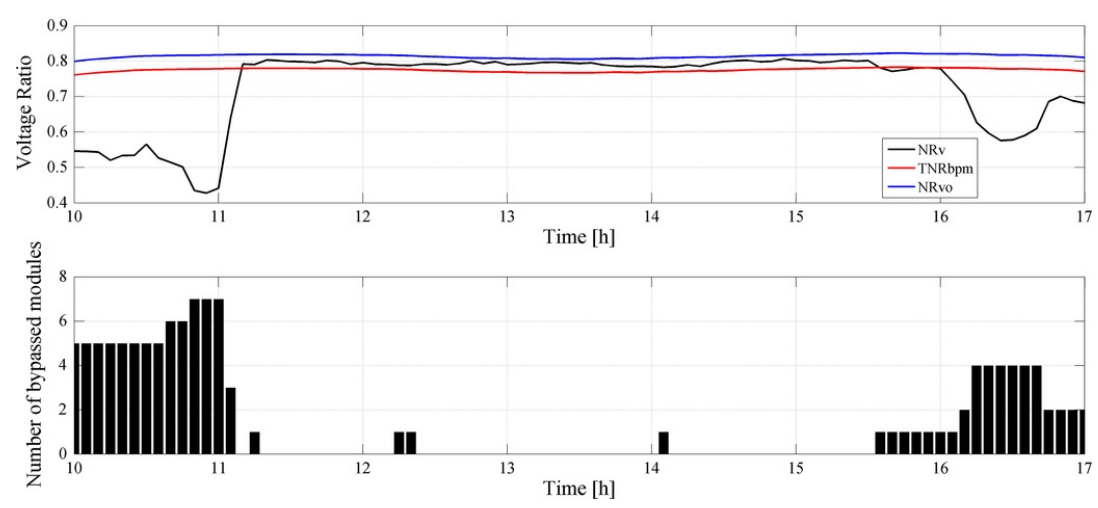

Fig. 10 Sub-generator 2: Voltage ratio and number of bypassed modules.

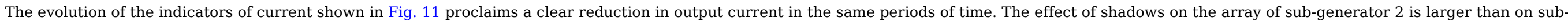
generator 1 in both cases: Output voltage and current, as it might be expected.

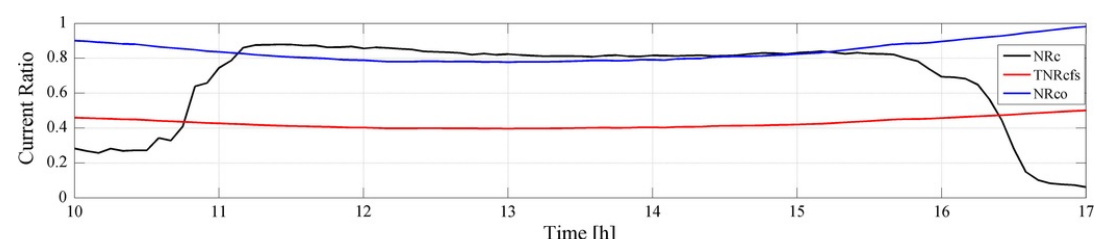

Time $[\mathrm{h}$

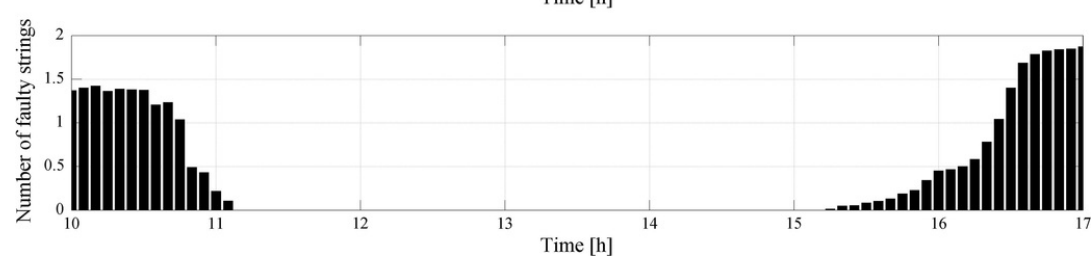

Fig. 11 Sub-generator 2: Current Ratio and equivalent number of faulty strings.

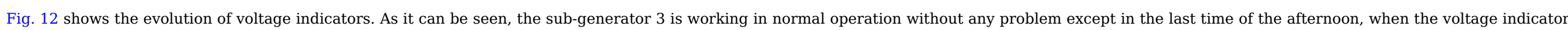
$N R v$, appears below the threshold TNRbpm and it seems to be two bypassed modules in the string. The rest of the day there is no reduction in output voltage due to shadows on the array. 

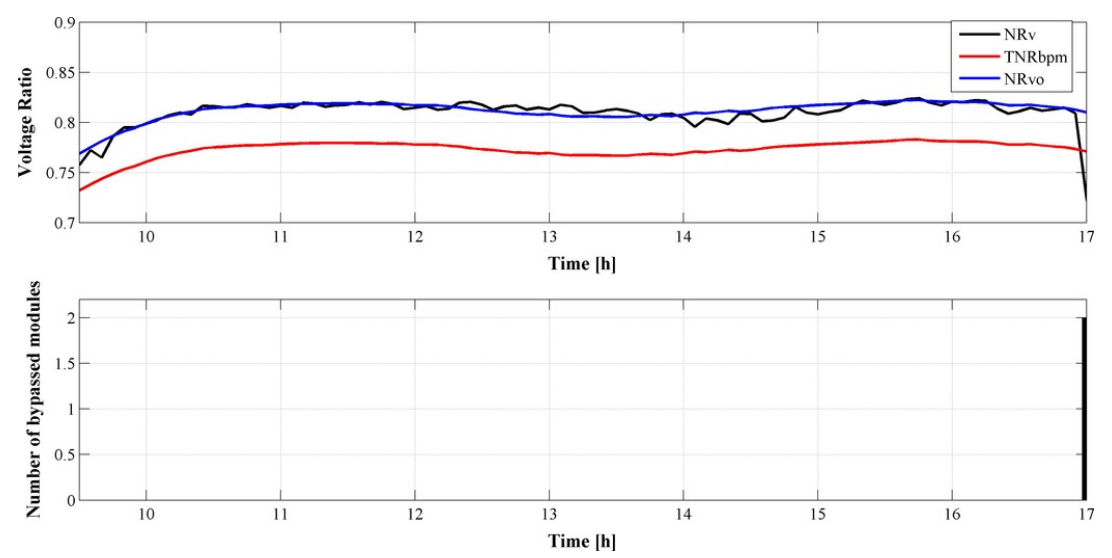

Fig. 12 Sub-generator 3: evolution of the voltage ratios and number of bypassed modules present in the array

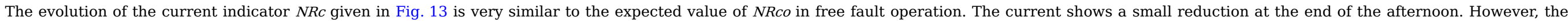
indicator of current, $N R c$, remains over the corresponding threshold, TNRcfs, and no faulty strings are observed throughout the day.
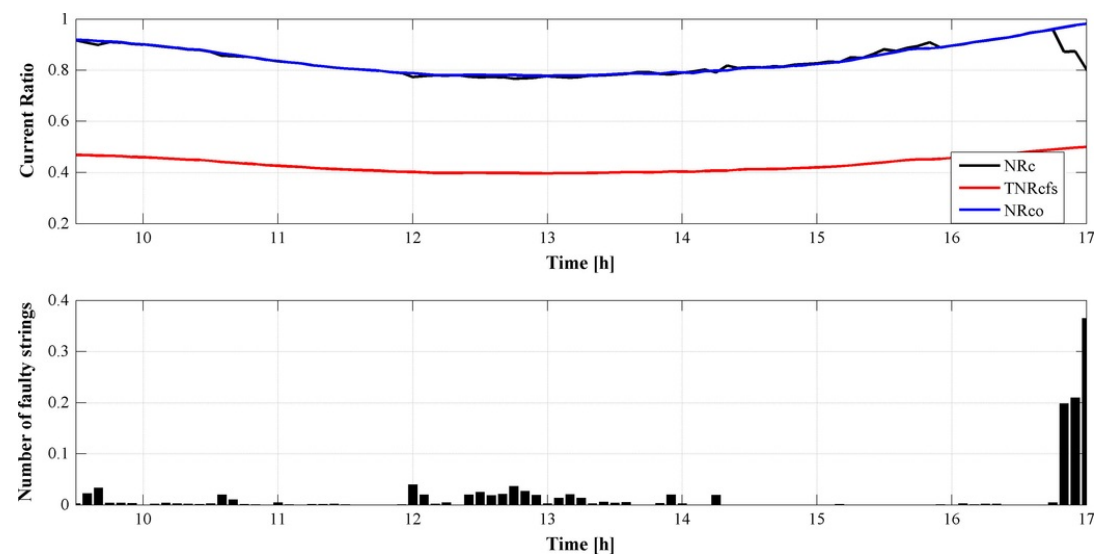

Fig. 13 Sub-generator 3: Current ratio and equivalent number of faulty strings.

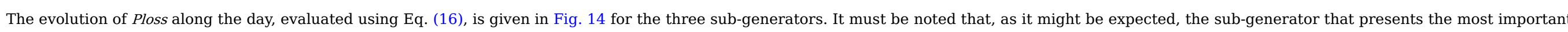

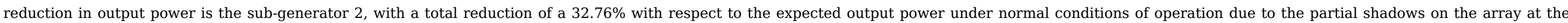

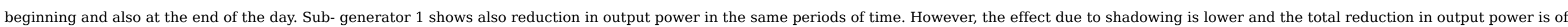
a $21.41 \%$ with respect to the expected one. Finally, sub-generator 3 is the array showing the lowest power losses. 


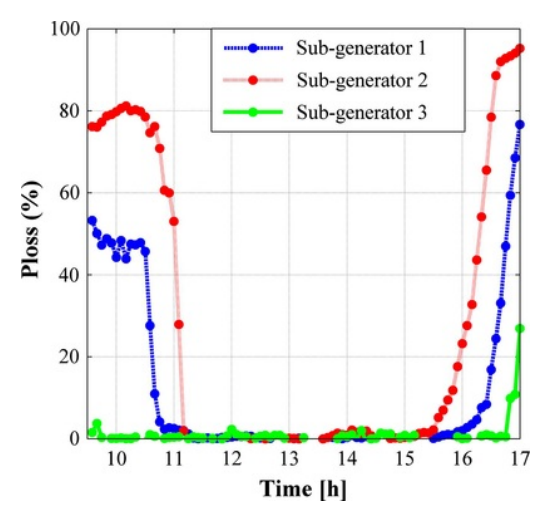

Fig. 14 Estimated power losses.

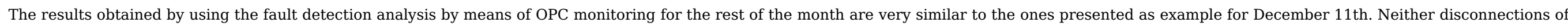

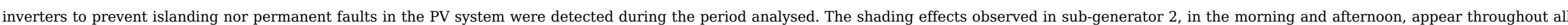

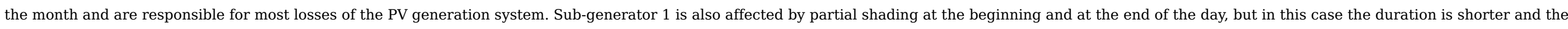

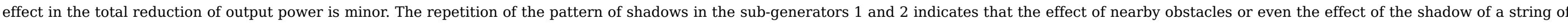
PV modules on another string is its most likely origin. Finally, sub-generator 3 presents the best behaviour and daily yields, being the least affected by shadows.

\section{Conclusions}

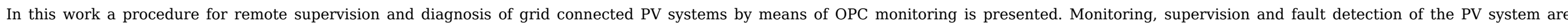
integrated in the same environment.

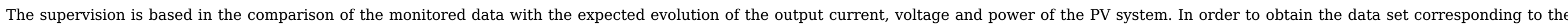

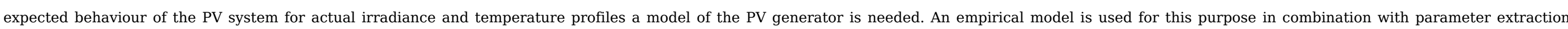

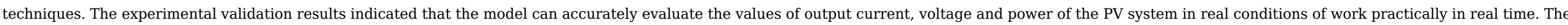
RMSE between real monitored data and results obtained from the modelling of the PV array were below $3.6 \%$ for all parameters even in cloudy days.

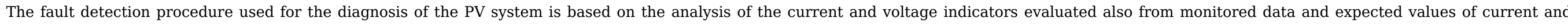

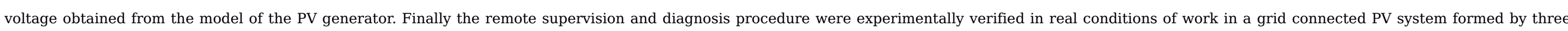

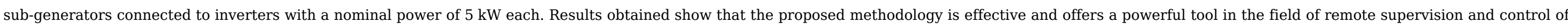
PV systems connected to the grid.

\section{References}

Alan Gordon. Programación COM y COM+. Anaya Multimedia, 2001.

Chine W., Mellit A., Pavan A.M. and Kalogirou S.A., Fault detection method for grid connected photovoltaic plants, Renew. Energy 66, 2014, 99-110.

Chouder A. and Silvestre S., Automatic supervision and fault detection of PV systems based on power losses analysis, Energy Convers. Manage. 51, 2010, 1929-1937.

Coleman A. and Zalewski J., Intelligent fault detection and diagnostics in solar plants, In: Intelligent Data Acquisition and Advanced Computing Systems (IDAACSs), 2011, 948-953.

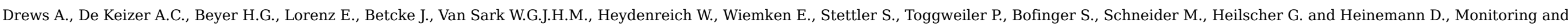
remote failure detection of grid connected PV systems based on satellite observations, J. Sol. Energy 81, 2007, 548-564. 
Firth S.K., Lomas K.J. and Rees S.J., A simple model of PV system performance and its use in fault detection, Sol. Energy 84, 2010, 624-635.

Holley D.W., Understanding and using OPC maintenance and reliabi- lity applications, Comput. Contr. Eng. J. 15 (1), 2004, $28-31$.

Jarque C.M., Bera A. and Anil K., A test for normality of observations and regression residuals, Int. Stat. Rev. 55 (2), 1987, 163-172.

King D.L., Kratochvil J.A. and Boyson W.E., Photovoltaic Array Performance Model, 2004, Department of Energy; USA.

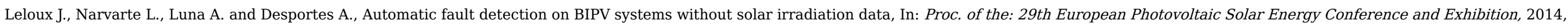
$1-7$.

Liu J., Lim K.W., Ho W.K., Tan K.C., Tay A. and Srinivasan R., Using the OPC standard for real-time process monitoring and control, IEEE Softw. 22 (6), 2005 , 54-59.

Martínez-Marchena I., Marco de trabajo para la generación de software para la gestión de sistemas de energía solar, Universidad de Málaga, PhD Thesis2015, Universidad de Málaga, junio.

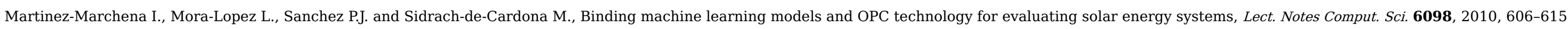

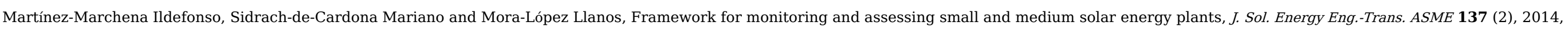

021007.

Peng J., Lu L., Yang H. and Ma T., Validation of the Sandia model with indoor and outdoor measurements for semi-transparent amorphous silicon PV modules, Renew. Energy 80, 2015, 316-323.

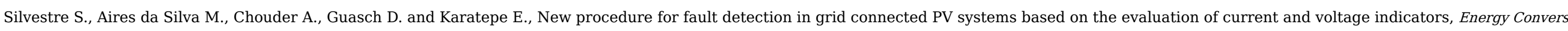
Manage. 86, 2014, 241-249.

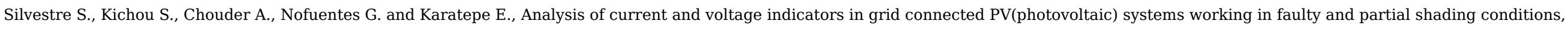

Energy 86, 2015, 42-50.

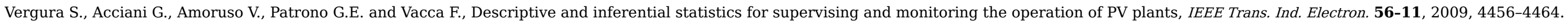

Wu Y., Lan Q. and Sun Y., Application of BP neural network fault diagnosis in solar photovoltaic system, In: Mechatronics and Automation (ICMA), 2009, 2581-2585.

\section{Highlights}

- Standard OPC for monitoring and supervision of grid connected PV systems is applied.

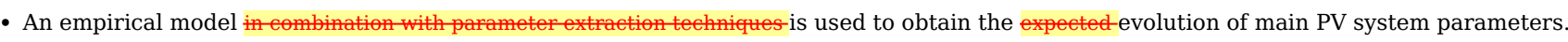

- The RMSE

- A powerful tool for remote supervision and control of PV systems isented.

\section{Queries and Answers}

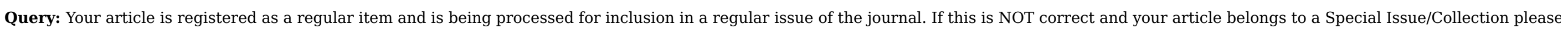
contact j.aranha@elsevier.com immediately prior to returning your corrections.

Answer: It is correct

Query: The author names have been tagged as given names and surnames (surnames are highlighted in teal color). Please confirm if they have been identified correctly. 


\section{Answer: The surnames are correct}

Query: Highlights should only consist of 85 characters per bullet point, including spaces. The highlights provided are too long; please edit them to meet the requirement. Answer: The highlights have been modified.

Query: Please note that the reference style has been changed from a Numbered style to a Name-date style as per the journal specifications.

Answer: OK 